\title{
SPECKLE REDUCTION IN SAR IMAGES USING A BAYESIAN MULTISCALE APPROACH
}

\author{
F. Zakeri ${ }^{1}$, M. R. Saradjian ${ }^{1 *}$, M. R. Sahebi ${ }^{2}$ \\ ${ }^{1}$ School of Surveying and Geospatial Engineering, College of Engineering, University of Tehran, \\ Tehran 1439957131, Iran - (fatemeh.zakeri, sarajian)@ut.ac.ir \\ ${ }^{2}$ Photogrammetry and Remote Sensing Department, K. N. Toosi University of Technology, Tehran 1969764499, Iran- \\ sahebi@kntu.ac.ir
}

KEYWORDS: Curvelet Transform, SAR Images, Speckle Noise, Statistical Modeling

\begin{abstract}
:
Synthetic aperture radar (SAR) images are corrupted by speckles, which influence the interpretation of the images. Therefore, to reduce speckles and obtain reliable information from images, researchers studied different methods. This study proposes a Bayesian multiscale method, to reduce speckles in SAR images. First, it was shown that Laplacian probability density function can capture the characteristics of noise-free curvelet coefficients, and then, a maximum a posteriori (MAP) estimator was designed for estimating them. Comparison of the results obtained with those obtained from conventional speckle filters, such as Lee, Kuan, Frost, and Gamma filters, and also curvelet non-Bayesian despeckling, shows better achievement of the proposed algorithm. For instance, the improvement in different parameters is as follows: 'noise mean value' (NMV) 0.24 times, 'noise standard deviation' $(N S D) 0.34$ times, 'mean square difference' $(M S D) 2.6$ times and 'equivalent number of looks' $(E N L) 0.61$ times.
\end{abstract}

\section{INTRODUCTION}

Synthetic Aperture Radar (SAR) images, because of their abilities, like all-weather and day-and-night capturing, have many applications, such as monitoring of agriculture, and mapping of floods, forest, snow, and wetland. But, they contain speckles resulting from the coherent nature of incident energy. The speckled nature of the images reduces the quality of interpreting radar data (Richards, 2009).

Although traditional filtering-based methods, such as those of Lee (Lee, 1980), Kuan et al.(Kuan, Sawchuk, Strand, \& Chavel, 1985), and Frost et al. (Frost, Stiles, Shanmugan, \& Holtzman, 1982) generally perform well in reducing speckle, but most of the images still contain blurred features and edges (Gagnon \& Jouan, 1997). Therefore, speckle reduction, on multiscale, has been studied by many researchers over the last few decades (Zong, Laine, \& Geiser, 1998), (Xie, Pierce, \& Ulaby, 2002), and (Argenti \& Alparone, 2002).

Curvelet, with its directional sensitivity and much better preserved edges than those of other multiscale transforms, such as wavelet, is useful for image-processing (Candes, Demanet, Donoho, \& Ying, 2006). The curvelet-based denoising methods have been introduced recently (Starck, Candès, \& Donoho, 2002), (Amirmazlaghani \& Amindavar, 2010), (Ali, Swami, \& Singhai, 2010), (Li, Gong, Feng, \& Zhang, 2011), and (Zakeri \& Zoej, 2015).

Denoising in the curvelet domain involves three major steps. First, curvelet transform of the noisy image is calculated; second, the noisy coefficients are modified; third, the inverse transform is taken to obtain the denoised one.

For the second step, two major approaches are available: 1)Thresholding technique (Starck et al., 2002), (Ali et al., 2010), (Li et al., 2011); 2) Bayesian estimation shrinkage technique (Amirmazlaghani \& Amindavar, 2010). Thresholding technique needs a correctly chosen threshold value to achieve good results. The main idea of the Bayesian method is to model curvelet coefficient with prior probability distributions and then estimate the noise-free coefficients. Estimating threshold and determining thresholding rules are the two drawbacks of the thresholding technique. Therefore, in this study, a new Bayesian multiscale speckle reduction approach is proposed. In the proposed algorithm, by taking the logarithm of the original data, a multiplicative speckle phenomenon is first transformed into an additive one. Then, curvelet transform is applied to the transformed image. Subsequently, Laplacian probability distribution function (pdf) is used to model noise-free coefficients and to derive corresponding shrinkage function by using Bayesian estimation theory. Then, this shrinkage function is used to modify curvelet coefficients. Finally, the denoised image is obtained by applying the inverse curvelet transform and exponential transform to the image.

\section{PROPOSED METHOD}

Speckle has multiplicative nature that is transformed into a Gaussian additive one by taking the logarithm of the original image (Arsenault \& April, 1976). Based on the Gaussianity assumption for log-transformed SAR image, the denoising of an image corrupted by white Gaussian noise, in the curvelet domain, will be considered, i.e.,

$$
y=x+n
$$

where, $y$ and $x$ are the noisy curvelet coefficient and the corresponding noise-free coefficient, respectively. Also, $n$ represents the additive Gaussian noise coefficient. The aim of denoising is to estimate noise-free coefficient, i.e. $x$ from noisy coefficient $y$. For achieving this, the maximum a posteriori (MAP) estimator will be used, which can be defined by

$$
\hat{x}=\arg \max p_{x \mid y}(x \mid y)
$$

By using Bayes rule, Equation (3) can be obtained by

$$
\hat{x}(y)=\underset{x}{\arg \max }\left[p_{n}(y-x) \cdot p_{x}(x)\right]
$$

Equation (3) is equivalent to

$$
\hat{x}(y)=\arg \max \left[\log \left(p_{n}(y-x)\right)+\log \left(p_{x}(x)\right)\right]
$$

\footnotetext{
* Corresponding author
} 
For solving equation (4), noise PDF and noise-free coefficients PDF are needed. Noise PDF can be expressed from the assumption as zero-mean Gaussian with variance $\sigma_{n}$, i.e.,

$$
p_{n}(n)=\frac{1}{\left(2 \pi \sigma_{n}^{2}\right)^{\frac{1}{2}}} \cdot \exp \left(-\frac{n^{2}}{2 \sigma_{n}^{2}}\right)
$$

Also, $p_{x}(x)$ is assumed to be a Laplacian density with variance $\sigma^{2}$, i.e.,

$$
p_{x}(x)=\frac{1}{b \sigma} \exp \left(-\frac{|b x|}{\sigma}\right)
$$

where $b$ is a scale parameter.
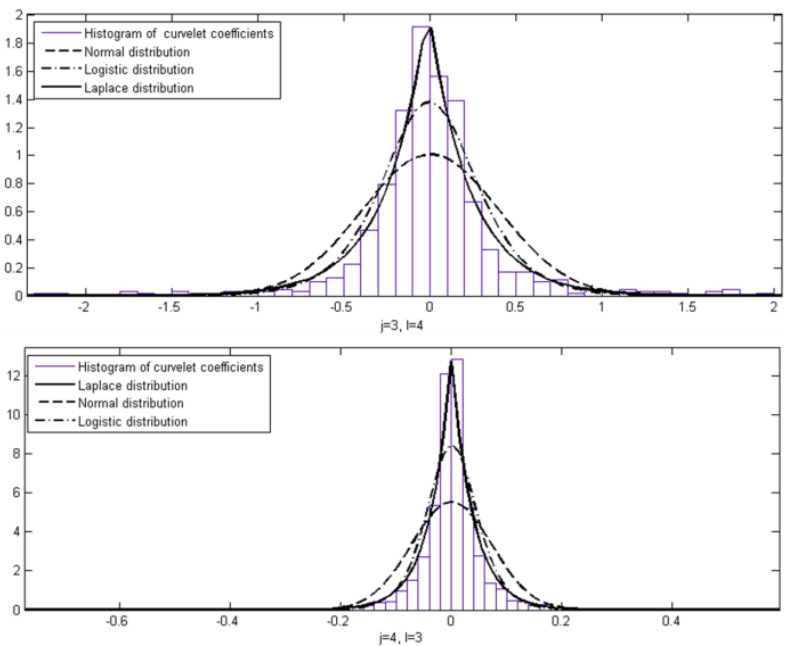

Figure. 1: The histogram of the curvelet coefficients for noisefree data sets (Scale index $j$ and orientation index $l$ ).

(Figure. 1) illustrates the histogram of the curvelet coefficients at two different scales and orientations, computed from noise-free images. Compared with Normal and Logistic distributions, it is clear from figure 1 that the Laplacian distribution fits better into them.

After some manipulation, the MAP estimator of $x$ is derived as

$$
\hat{x}(y)=\operatorname{sign}(y)\left(\left|y-\frac{b \sigma_{n}^{2}}{\sigma}\right|\right)_{+}
$$

Here $(x)_{+}$is defined as $\max (x, 0)$. For solving equation (7), three unknown parameters i.e. $b, \sigma$ and $\sigma_{n}$ must be estimated. To estimate the noise variance $\sigma_{n}^{2}$ from the noisy curvelet coefficients, a robust median estimator and Monte Carlo simulation (Starck et al., 2002), as well as Arithmetic and Geometric means, are used as follows:

$$
\sigma_{n}^{2}=\left(\beta * \sigma_{j l}\right)+|A M-G M|_{j l}
$$

where, $\sigma_{s d}$ is the average energy distribution of noise in curvelet coefficients at scale $j$ and orientation $l$ that can be estimated by

Monte Carlo simulation. Also, $\beta$ can be estimated by the median estimator from the finest scale curvelet coefficients. $A M$ and $G M$ are the Arithmetic and Geometric means at each scale $j$ and

orientation $l$. In order to adapt $\sigma_{n}$ much more with coefficients attributes at each scale and orientation, $A M$ and $G M$ are used.

The parameter $\sigma$ will be empirically estimated by using the neighboring coefficients in the region $N$ by

$$
\sigma=\sqrt{\max \left(\left(\frac{\sqrt{3}}{M} \sum_{y \in N}|y|\right)^{2}-\sigma_{n}^{2}, 0\right)}
$$

where, $M$ is the size of the neighborhood $N$, which is equal to a $3 \times 3$ squared window.

The parameter $b$ is equal to 3 for the first scale while $b=2$ for the others.

\section{EXPERIMENTAL RESULTS AND ANALYSIS}

The proposed method was applied to two real SAR data sets. A RADARSAT image from QUEBEC with $\mathrm{HH}$ polarization is the first image and the second one is an ENVISAT image from Montreal with VV polarization.

To evaluate noise reduction in real SAR images, in this paper, four parameters, namely $N M V$ ('noise mean value'), $N S D$ ('noise standard deviation'), $M S D$ ('mean square difference'), and $E N L$ ('equivalent number of looks'), were used and they are defined as follows (Mario Mastriani, 2006), (M Mastriani \& Giraldez, 2004):

$$
\begin{gathered}
N M V=\sum_{i=0}^{N-1} \hat{S}_{i} / N \\
N S D=\sqrt{\sum_{i=0}^{N-1}\left(\hat{S}_{i}-N M V\right)^{2} / N} \\
M S D=\sum_{i=0}^{N-1}\left(S_{i}-\hat{S}_{i}\right)^{2} / N \\
E N L=N M V^{2} / N S D^{2}
\end{gathered}
$$

$N S D$ and $N M V$ show the content of the speckle; so, smaller NSD and $N M V$ imply that the denoising produced a clearer image. $E N L$ shows the ability of different filters to suppress speckle noise in homogeneous areas, and $M S D$ is square of the difference between the SAR image and the filtered image; therefore, bigger $E N L$ and larger MSD imply better noise reduction (Mario Mastriani, 2006), (M Mastriani \& Giraldez, 2004).

ENL should be measured over a uniform image region (M Mastriani \& Giraldez, 2004). Owing to the difficulty in recognizing uniform regions, the image was divided into smaller regions of $25 \times 25$ pixels, and the $E N L$ values obtained in each area were averaged to calculate $E N L$ criterion.

Criteria for real SAR images were obtained on a subset of these images. (Table. 1) and (Table. 2) summarize the assessment parameters. 
Table. 1: Speckle noise reduction results of ENVISAT

\begin{tabular}{ccccc}
\hline & $\boldsymbol{N M} \boldsymbol{V}$ & $\boldsymbol{N S \boldsymbol { D }}$ & $\boldsymbol{M S D}$ & $\boldsymbol{E N L}$ \\
\hline Proposed & 147722.5 & 84177.52 & $9.28 \mathrm{e}+09$ & 1.15 \\
\hline Frost & 193975.6 & 124031.6 & $3.76 \mathrm{e}+09$ & 0.97 \\
\hline Gamma & 173423.7 & 127937 & $2.56 \mathrm{e}+09$ & 0.75 \\
\hline Kuan & 195656.3 & 122792.2 & $4.13 \mathrm{e}+09$ & 1.04 \\
\hline Lee & 188949.9 & 125681.9 & $2.91 \mathrm{e}+09$ & 0.92 \\
\hline K-sigma & 164529.6 & 128170.3 & $4.3 \mathrm{e}+09$ & 0.71 \\
\hline
\end{tabular}

Table. 2: Speckle noise reduction results of RADARSAT

\begin{tabular}{ccccc}
\hline & $\boldsymbol{N M} \boldsymbol{V}$ & $\boldsymbol{N S \boldsymbol { D }}$ & $\boldsymbol{M S D}$ & $\boldsymbol{E N L}$ \\
\hline Proposed & 1599.386 & 284.9263 & 52678.6 & 10.26 \\
\hline Frost & 1660.946 & 288.6847 & 49325.99 & 8.71 \\
\hline Gamma & 1649.483 & 297.0648 & 47738.29 & 8.57 \\
\hline Kuan & 1659.175 & 289.1015 & 50188.42 & 8.70 \\
\hline Lee & 1657.486 & 291.2301 & 49093.56 & 8.63 \\
\hline K-sigma & 1614.675 & 362.3948 & 33197.89 & 6.82 \\
\hline
\end{tabular}

It is obvious from these Tables that the proposed method, owing to a sparse representation of the image and edge-preserving ability of the curvelet transform, together with prior knowledge about estimating noise-free coefficient, obtained better results than other speckle-reduction methods.

(Figure. 2(a)-(h)) and (Figure. 3(a)-(h)) show a subset of RADARSAT and ENVISAT images and their denoised images, obtained by the filters mentioned and the proposed method. Figures. 2 \& 3, and (Table. 1) \& (Table. 2) show that the proposed method, in comparison with other filters, removed noise while preserving important details (edges and features).

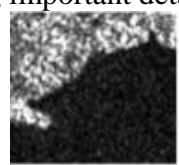

(a)

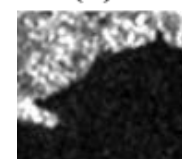

(d)

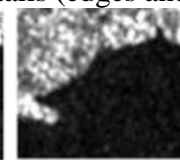

(b)

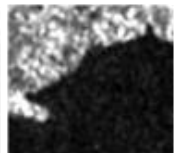

(e)

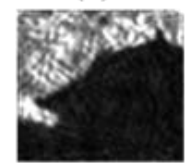

(g)

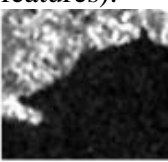

(c)

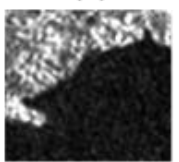

(f)

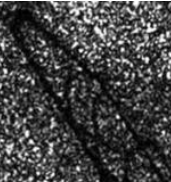

(a)

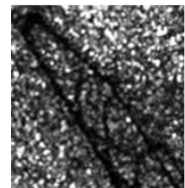

(d)

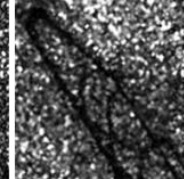

(b)

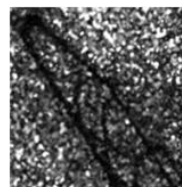

(e)

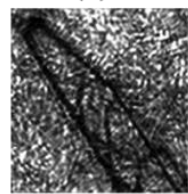

(g)

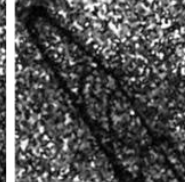

(c)

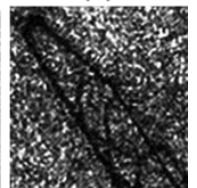

(f)

Figure. 3: Despeckling results of different filters for ENVISAT image: (a) ENVISAT Image, (b) Frost, (c) Gamma, (d) Kuan, (e) Lee, (f) K-sigma, (g) Proposed method.

\section{CONCLUSION}

A new speckle-reduction method, using curvelet transform and Bayesian estimation shrinkage technique simultaneously, was presented. Curvelet transform is good in preserving the geometrical characteristics of an image, such as edges, which makes it suitable for image processing purposes, like noise reduction. After studying curvelet noise-free coefficients, it was shown that the Laplacian probability density function can capture the characteristics of noise-free curvelet coefficients. Then, a maximum a posteriori (MAP) processor was designed for estimating noise-free curvelet coefficients. The proposed method was tested on real SAR images, and it was shown that, in comparison with other commonly used filters, like Lee, Frost, Gamma, and Kuan filters and also curvelet non-Bayesian despeckling, the proposed method provides much better results in both edge preservation and speckle noise reduction. Improvement in terms of different parameters is as follows: $N M V$ 0.24 times, NSD 0.34 times, $M S D 2.6$ times and ENL 0.61 times. Besides, the proposed method does not need to compute thresholding value; further, it is a low-complexity image denoising algorithm.

\section{REFERENCES}

Ali, A.-D., Swami, P. D., \& Singhai, J. (2010). Modified Curvelet Thresholding Algorithm for Image Denoising. Journal of Computer Science, 6(1).

Amirmazlaghani, M., \& Amindavar, H. (2010). Two novel Bayesian multiscale approaches for speckle suppression in SAR images. Geoscience and Remote Sensing, IEEE Transactions on, 48(7), 2980-2993.

Argenti, F., \& Alparone, L. (2002). Speckle removal from SAR images in the undecimated wavelet domain. Geoscience and Remote Sensing, IEEE Transactions on, 40(11), 2363-2374.

Arsenault, H., \& April, G. (1976). Properties of speckle integrated with a finite aperture and logarithmically transformed. JOSA, 66(11), 1160-1163. 
Candes, E., Demanet, L., Donoho, D., \& Ying, L. (2006). Fast discrete curvelet transforms. Multiscale Modeling \& Simulation, 5(3), 861-899.

Frost, V. S., Stiles, J. A., Shanmugan, K. S., \& Holtzman, J. (1982). A model for radar images and its application to adaptive digital filtering of multiplicative noise. Pattern Analysis and Machine Intelligence, IEEE Transactions on(2), 157-166.

Gagnon, L., \& Jouan, A. (1997). Speckle filtering of SAR images: a comparative study between complex-wavelet-based and standard filters. Paper presented at the Optical Science, Engineering and Instrumentation'97.

Kuan, D. T., Sawchuk, A. A., Strand, T. C., \& Chavel, P. (1985). Adaptive noise smoothing filter for images with signaldependent noise. Pattern Analysis and Machine Intelligence, IEEE Transactions on(2), 165-177.

Lee, J.-S. (1980). Digital image enhancement and noise filtering by use of local statistics. Pattern Analysis and Machine Intelligence, IEEE Transactions on(2), 165-168.

Li, Y., Gong, H., Feng, D., \& Zhang, Y. (2011). An adaptive method of speckle reduction and feature enhancement for SAR images based on curvelet transform and particle swarm optimization. Geoscience and Remote Sensing, IEEE Transactions on, 49(8), 3105-3116.

Mastriani, M. (2006). Fuzzy Thresholding in Wavelet Domain for Speckle Reduction in Synthetic Aperture Radar Images. International Journal of Intelligent Technology, 1(3).

Mastriani, M., \& Giraldez, A. (2004). Enhanced directional smoothing algorithm for edge-preserving smoothing of synthetic-aperture radar images. Journal of Measurement Science Review, 4(3), 1-11.

Richards, J. A. (2009). Remote sensing with imaging radar. New York City: Springer.

Starck, J.-L., Candès, E. J., \& Donoho, D. L. (2002). The curvelet transform for image denoising. Image Processing, IEEE Transactions on, 11(6), 670-684.

Xie, H., Pierce, L. E., \& Ulaby, F. T. (2002). SAR speckle reduction using wavelet denoising and Markov random field modeling. Geoscience and Remote Sensing, IEEE Transactions on, 40(10), 2196-2212.

Zakeri, F., \& Zoej, M. J. V. (2015). Adaptive method of speckle reduction based on curvelet transform and thresholding neural network in synthetic aperture radar images. Journal of Applied Remote Sensing, 9(1), 095043.

Zong, X., Laine, A. F., \& Geiser, E. A. (1998). Speckle reduction and contrast enhancement of echocardiograms via multiscale nonlinear processing. Medical Imaging, IEEE Transactions on, $17(4), 532-540$. 